Article

\title{
Public Willingness to Pay for Transforming Jogyesa Buddhist Temple in Seoul, Korea into a Cultural Tourism Resource
}

\author{
Seul-Ye Lim, Ho-Young Kim and Seung-Hoon Yoo * \\ Department of Energy Policy, Graduate School of Energy \& Environment, \\ Seoul National University of Science \& Technology, 232 Gongreung-Ro, Nowon-Gu, Seoul 01811, Korea; \\ sylim@seoultech.ac.kr (S.-Y.L.); sksflyh@seoultech.ac.kr (H.-Y.K.) \\ * Correspondence: shyoo@seoultech.ac.kr; Tel.: +82-2-970-6802
}

Academic Editor: Ian Patterson

Received: 23 May 2016; Accepted: 30 August 2016; Published: 6 September 2016

\begin{abstract}
The Jogyesa Buddhist Temple (JBT), located in Seoul, Korea, is the chief temple of the Jogye Order, which represents Korean Buddhism. The Seoul government plans to transform the JBT into a cultural tourism resource and a historical site. This study attempts to analyze the willingness to pay (WTP) for implementing the transformation, which includes building a new shopping arcade for Buddhist culture and tourism, constructing a museum for the teaching of history and an experience center for Korean traditional culture in the precincts of JBT, and making an open space for domestic and/or foreign visitors. To this end, the study looks into the WTP for the implementation, reporting on a contingent valuation $(\mathrm{CV})$ survey that was conducted with 500 Seoul households. The single-bounded dichotomous choice CV model and a spike model were applied to derive the WTP responses and analyze the WTP data with zero observations, respectively. The mean yearly WTP was computed to be KRW 7129 (USD 6.30) per household for the next five years, with the estimate being statistically significant at the $1 \%$ level. Expanding the value to the Seoul population gives us KRW 25.4 billion (USD 22.5 million) per year. The present value of the total WTP amounts to KRW 114.6 billion (USD 101.3 million) using a social discount rate of $5.5 \%$. We can conclude that Seoul households are ready to shoulder some of the financial burden of implementing the transformation.
\end{abstract}

Keywords: Jogyesa Buddhist Temple; contingent valuation; willingness to pay; spike model

\section{Introduction}

Buddhism, a word meaning the doctrine explained by Buddha and the way to become a Buddha, is one of the major religions of the world [1,2]. Buddhism was first introduced to Korea at the end of the 4th century, in 372, which is about 800 years after the death of the historical Buddha. At that time, shamanism was the indigenous religion in what was then Korea. Buddhism became mixed with shamanism in harmony with the rites of nature worship. Thus, Korean Buddhism came to have its own features and its roots went deep into the Korean soil. Currently, about a quarter of the population of Korea are Buddhists.

Jogyesa Buddhist Temple (JBT) was established in 1395, at the start of the Chosun Dynasty. JBT was originally built in 1910 by monks longing for the independence of Korean Buddhism and the recovery of Koreans' self-esteem, and it bore the name of Gakhwangsa Buddhist Temple. In 1937, it was moved to its current location where it was reconstructed. The construction was completed in 1938. At this time, the temple was known as Taegosa. This was during the Japanese occupation of Korea (1910-1945). After liberation, in 1954, the Buddhist Purification Movement was established. The goal of the movement was to eliminate any Japanese influence remaining from the Japanese 
occupation. In 1954, the temple was renamed Jogyesa. This was a way for Korea to move on and forget the past horrors of the Japanese occupation [3].

Since 1936, JBT has been the head temple of the first district of the Jogye Order of Korean Buddhism. JBT is the most important, popular, and representative Buddhist temple in Korea. JBT was the first propagation center to argue for the legitimacy of local Buddhism and the first temple located inside the boundary of the four gates of the city of Seoul, the capital of Korea. The Daeungjeon (Main Dharma Hall) of JBT is the largest temple building in the city of Seoul and it, alone, is a spectacular and colorful sight for any visitor. Visitors are surprised to find a beautiful setting and sense of calmness in the middle of the hectic and crowded city. The grounds are a great escape from the big city for both locals and tourists. Some natural elements that should not be missed include a rare 500 year old Baeksong lacebark pine tree and a 450 year old Chinese scholar tree. JBT may not have the most beautiful or grand grounds in Korea, but its strength lies in its convenient location, which makes it one of the most visited and popular temples in central Seoul [3].

JBT promotes the embodiment of a society where people live together, and Bodhisattva's spirit. Moreover, JBT is a historic site that has survived the turbulent modern history of Korea. JBT is located within the heart of Seoul, where the public can enjoy rest and leisure. JBT has positioned itself well as the chief temple of the Order through the remodeling of the Main Hall and the construction of the One Pillar Gate. It is also a leader in devotional practices and religious and public welfare activities. The Main Hall is open $24 \mathrm{~h}$ a day to all members of the public, allowing both local people and foreigners to visit. JBT promotes itself as an open space, inviting everyone to the temple's events and ceremonies [4].

However, JBT has a painful recent past. A military coup took place against the ruling civilian government, and a military dictatorship came into power in Korea in 1980. In order to strengthen the dictatorship, the dictator arrested 153 monks affiliated with the Jogye Order by force on 27 October 1980, and used violence and torture against them. We call this incident the "10.27 event". The people worked hard for democracy and initiated a civilian government in 1987. The Korean government investigated the 10.27 event, officially announced that it was a clear abuse of state power, and promised to rehabilitate the victims' reputations and pay compensation for the damage caused [5].

The Seoul city government is planning to build a museum for teaching history, particularly the history of the 10.27 event, in the precincts of JBT. There are also plans to build a new shopping arcade for Buddhist culture and tourism, to construct an experience center for Korean traditional culture, and to make an open space for domestic and/or foreign visitors. The Seoul city government's purpose is to transform JBT into a cultural tourism resource and historical site. The government should make an informed decision on whether or not to implement the transformation. If the transformation goes ahead, the people of Seoul will absorb the costs through local taxes. Current and future generations will enjoy the benefits of the transformation [6].

The JBT is a famous and representative cultural and historical site located on Seoul, the capital of Korea, but it suffers from far too run-down facilities for convenience and insufficient open space for visitors. For the sake of JBT's sustainability, remodeling, or transforming JBT into a cultural tourism resource is widely demanded by the Seoul residents, domestic and foreign visitors, and policy-makers of the Seoul government. However, considerable public expenditures are required to do so. Thus, public support for the transformation is inevitably needed to justify the expenditures. This study tries to analyze the degree of the public support to provide responsible quantitative information on the public WTP for the transformation with policy-makers. We think that this is an interesting feature of our study.

When economic efficiency is employed as the sole criterion, one should perform a conventional cost-benefit analysis and determine whether or not to implement the transformation [7]. The costs of implementing the transformation can be easily measured against the benefits. On the other hand, assessing the economic benefits arising from this transformation is quite complicated. The economic benefits are the sum of its public and private values. Therefore, this paper attempts to assess the public 
value of the transformation by investigating the willingness to pay (WTP) for the transformation so as to obtain quantitative information that can be provided to policy-makers.

To this end, we use the contingent valuation (CV) technique. As will be discussed in the next section, this has been most widely employed in the context of empirical works measuring the economic value of a cultural or historical good [8-10]. The CV method usually asks randomly-selected respondents questions about whether they are willing to pay a specified amount for obtaining a good, with a survey of randomly selected households [11-14]. A large number of respondents in our CV survey revealed that they were unwilling to pay for the transformation, as will be explained below. In other words, we obtained WTP data with observations of zero. To reflect these data in the analysis, we utilized a spike model that can explicitly incorporate the possibility of zero WTP responses as well as positive WTP responses.

Therefore, the purpose of this paper is to measure the public value of transforming JBT in Seoul, Korea into a cultural tourism resource through analyzing the WTP for the transformation, using the $\mathrm{CV}$ method. The remainder of the paper is structured as follows: some methodological issues are explained in Section 2; the WTP model and the data used here are described in Section 3; the estimation results are presented in Section 4; and the final section concludes the paper.

\section{Methodological Issues}

\subsection{Method of Deriving the WTP for Transforming JBT into a Cultural Tourism Resource}

As addressed above, the economic benefits of a project are the sum of the public and private values of a project [15]. The private value is usually estimated with ease, but the public value is hard to measure. The public value can be assessed using a stated preference technique, such as the CV method. The CV technique has been widely applied in the literature for obtaining the WTP for a non-market good. There are no restrictions on the object to be valued when using the CV method. In particular, it is especially useful because it can capture the non-use or existence value of a good that cannot be measured through a market mechanism. Non-market goods include cultural goods or public goods, like the transformation. Thus, as explained earlier, this study seeks to utilize the CV approach to assess the WTP for implementing the transformation.

The CV method asks randomly chosen consumers a question concerning their WTP for the particular good, using a well-structured survey [16-20]. Some people may doubt the practicality and usefulness of the CV technique because it gathers information from a survey of respondents. In this regard, the blue-ribbon National Oceanic and Atmospheric Administration (NOAA) Panel came to the influential conclusion that the $\mathrm{CV}$ method can produce reliable quantitative information that can be utilized in decision-making with respect to both public administration and jurisdiction, provided that the guidelines proposed by the NOAA Panel are observed [21]. Moreover, following the guidelines can ensure the validity and accuracy of the CV method.

For example, the goods of concern should be familiar to the public, like the JBT which is familiar with citizen because it is representative of the Korea Buddhist temple and located in the center of Seoul, the CV survey should be administered through face-to-face interviews by professionally-trained interviewers rather than by telephone or mail interviews, a suitable payment vehicle should be adopted and presented to the respondents, and the substitutes to the goods should be explained to the respondents in the survey. These conditions were met in our study, as will be discussed in detail below.

\subsection{The Goods to Be Valued}

Located around the temple grounds are a variety of stores and shops that specialize in Buddhist items that can be great souvenirs. However, the buildings where the stores and shops are located and the environment surrounding JBT are now rather old-fashioned and somewhat run-down. Thus, it is necessary for a new shopping arcade for Buddhist culture and tourism to be built. The items 
for sale would include small Buddha statues, prayer beads, small wooden gongs, monks' clothing, and incense. Moreover, as explained above, constructing a museum to teach history (the 10.27 event) and an experience center for Korean traditional culture in the precincts of JBT, and making an open space for domestic and/or foreign visitors, are being considered. The Seoul government should decide whether to invest public money into the transformation, including these activities. We attempt to derive the WTP for the transformation.

\subsection{Survey Design Issues}

We commissioned a professional survey firm to arrange the CV field survey. The firm drew a random sample of 500 households from the Seoul population to obtain information on the households' WTP for the transformation and also information about their socioeconomic characteristics. The study area of this research was restricted to Seoul, the capital of Korea, where the population equals a quarter of the total national population. Seoul is a large city, with a population of around ten million people (3.5 million households). In order to draw a random sample of this population, sampling was conducted by a professional polling firm (Research Prime Service, Inc.) located on Seoul. Seoul comprises 25 wards (called gu). Our sample of 500 households was allocated to the wards or gu in proportion to each gu's population characteristics, such as age, income, gender, and education level, resulting in 15 to 25 households being assigned to each gu. The population characteristics come from 2010 "National Population and Housing Census" conducted by the Korea National Statistical Office.

A CV survey can be conducted face-to-face or by telephone or mail interviews. Of these, the response rate to a mail survey is usually quite low, and a telephone survey can present only a limited volume of information to the respondents. However in the CV survey, we wanted to convey a large amount of information explaining the transformation to the respondents, to use pictures to describe what the situation would be like with and without the transformation, and to outline the expected effects of the transformation. This is why we used face-to-face interviews.

We gave sufficient information to the interviewers about the purposes and background of the CV survey, and about how to answer questions raised by interviewees during the CV survey. When professional interviewers performed the CV survey, they showed exactly the information on the transformation with explanatory documents and visual materials to the respondents. The interviewers explained to respondents that store owners who are expected to be losers will be provided an arcade space in the basement of JBT and the arcade space will attract a number of visitors. In addition, they explained that JBT, citizens, and visitors are expected to be winners, gaining a resting place and beautiful view.

Thus, these factors should be considered to design CV survey. We described this information on explaining documents to estimate WTP of respondents. The supervisors of the survey company trained the interviewers to implement the CV survey persuasively and effectively in 2013. In order to get reliable and responsible findings from the respondents, heads of households or homemakers aged between 20 and 65 years old were selected and interviewed for the CV survey. Judging from the interviewers' comments, the respondents stated their WTP responses without great difficulty. The final number of observations to be analyzed in our study was 500. The survey instrument consisted of three parts. The first was the introductory section that explained general background information about the transformation to the respondents and then asked the respondents about their perceptions of the transformation. The scenario that would provide the goods to be valued by the public was, therefore, clearly explained. The second part included questions about WTP for implementing the transformation. The context of the transformation, to ensure that the WTP questions were plausible, understandable, and meaningful, was presented. The final part contained questions related to the household's socioeconomic variables. 


\subsection{Method to Elicit WTP}

Our study used a close-ended question format. This is preferred in the literature to open-ended questions [16] because a respondent is likely to show strategic behavior and have difficulty in giving a WTP response when an open-ended question is asked. In addition, the blue-ribbon panel's report [21] supported the use of close-ended questions rather than open-ended questions. In the close-ended format, a respondent is asked to state whether he/she would pay a given amount (a "bid") to obtain a specified improvement in the quantity or quality of the goods to be valued.

This close-ended question format is usually called a single-bounded dichotomous choice (DC) question format since it asks just one question. The double-bounded (DB) DC question format asks a follow-up question to identify the WTP, citing a higher amount when the first response is "yes" and a lower amount when it is "no" [22]. While the DB question format results in higher statistical efficiency, we do not use this format here because it increases the bias involved in WTP responses and, thus, Bateman et al. [23] and Carson and Groves [24] do not favor it.

The findings from focus group interviews of 30 persons allowed us to derive a list of seven bids to be presented to the respondents. The amounts were KRW 1000, 2000, 3000, 5000, 7000, 10,000, and 15,000 (USD 0.90, 1.80, 2.70, 4.40, 6.20, 8.80, and 13.30) per household per year. At the time of the survey, USD 1 was approximately equal to KRW 1131. A bid from the list was randomly offered to each respondent in the CV survey.

\subsection{Payment Vehicle}

A respondent may be embarrassed to be asked directly for his/her WTP for implementing a project. Thus, it is helpful to introduce into the $\mathrm{CV}$ survey questionnaire a medium for paying an amount, to get the respondent to reveal her/his true WTP. We usually call the medium a payment vehicle. Payment vehicles found in the literature include taxes, funds, donations, and expenditure. The respondents should feel at home with the payment vehicle, and the goods to be valued should have a clear connection with it. In this respect, a tax is the most frequently employed payment vehicle for eliciting WTP responses. There are two options: national and local taxes. Of the two, a local tax is related directly to the transformation. Furthermore, among the various types of local taxes, property tax is the one that is the most familiar to respondents. Therefore, property tax is employed in this study as the payment vehicle. WTP admission fees to visit part(s) of the attraction would not necessarily require a majority and would adhere to the "user pays" principle and might come up with different results.

Next, two points should be decided. One is the frequency of payment-whether it should be monthly, quarterly, or annually, or a one-off payment. Following Egan et al.'s [25] suggestion, we used an annual payment. The other point is the duration of the payment. We decided that this should be five years. In short, each respondent was asked whether she/he was willing to pay a specified amount annually through a higher property tax for the next five years. The WTP question was: "Is your household willing to pay a given amount for implementing the project of transforming JBT into a cultural tourism resource through an increase in the property tax for next five years, if the success of the project is guaranteed?"

Moreover, some additional statements concerning the payment were provided in the survey questionnaire. For example, the respondents were told the following: "If a majority of respondents refuse to pay the cost, the project cannot be implemented. However, if a majority of respondents accept the payment, the projected can be conducted. Please bear in mind that there are many other projects to be implemented by the Seoul government as well as the project". The reference to property taxes and the need for majority support suggests an "all or nothing situation" in which all will pay for the benefits to a smaller number of users. WTP for admission fees to visit part(s) of the attraction would not necessarily require a majority and would adhere to the "user pays" principle and might come up with different results. 


\section{Modeling of WTP Responses}

\subsection{Basic WTP Model}

There are two approaches to modeling WTP responses gathered from a DC CV survey: The utility difference approach suggested by Hanemann [26], and the WTP function approach proposed by Cameron and James [27]. The first specifies the utility difference, using a random utility maximization model, while the second specifies the WTP responses directly as a dependent variable. As pointed out by McConnell [28], the choice between the two is not an issue of right and wrong but rests on the researcher's preference, because the two approaches are dual in the context of economics. The literature shows that the first approach has been more frequently applied than the second. Thus, we adopt the utility difference approach in our study. The ratios of "yes" responses to each given bid are the basic input in the application of this method.

Through the process of utility maximization under an income constraint, a discrete choice response about whether to pay a specified amount for achieving a given environmental improvement or provision of a public good is derived from each respondent. The independent variables of the utility function, $U$, include the respondent's income, socioeconomic characteristics, perceptions about the good to be valued, and its provision state. The provision state of the good to be valued is $S$, whose value is one if the good is provided and zero otherwise. The respondent's income and the other factors that affect the respondent's utility are $M$ and $T$. Thus, the utility function is defined as:

$$
U=V(S, M ; T)+\omega
$$

where $V$ is the indirect utility function that we obtain by inserting the solution to the utility maximization problem into the objective function of the respondent's utility, and $\omega$ is a random component of the utility where the $\omega$ 's are independent and identically distributed random variables with zero means.

The respondent will maximize his/her utility by showing willingness to pay a presented bid, $B$, to obtain the good to be valued if:

$$
V(1, M-B ; T)+\omega_{1} \geq V(0, M ; T)+\omega_{0}
$$

Rearranging Equation (2) produces:

$$
V(1, M-B ; T)-V(0, M ; T) \geq \omega_{0}-\omega_{1}
$$

The left-hand side of Equation (3) is the utility difference, defined as $\Delta V$, which is the systematic and deterministic part, while the right-hand side is the non-systematic and random part. Let $\omega_{0}-\omega_{1}$ be $\theta$ and $H_{\theta}(\cdot)$ be the cumulative distribution function (cdf) of $\theta$. Using Equation (3), we can express the probability of an answer "yes" to a given bid as:

$$
\operatorname{Pr}\{\text { response is "yes" }\}=\operatorname{Pr}\{\Delta V(B) \geq \theta\}=H_{\theta}[\Delta V(B)]
$$

From a different perspective, we can introduce the WTP, $X$, as a random variable into the description of the probability of responding "yes" to a presented bid as follows:

$$
\operatorname{Pr}\{\text { response is "yes" }\}=\operatorname{Pr}\{X \geq B\} \equiv 1-F_{X}(B)
$$

where $F_{X}(\cdot)$ is the cdf of $X$. Thus, comparing Equations (4) and (5) yields:

$$
1-F_{X}(B)=H_{\theta}[\Delta V(B)]
$$


Therefore, we do not need to assume the functional form of $H_{\theta}(\cdot)$. All we have to do is to assume the functional form of $F_{X}(\cdot)$, and estimate its parameters. Usually, we assume that $\Delta V=\alpha-\beta B$ where $\lambda=(a, b)$ is a parameter vector to be estimated.

\subsection{Model for Dealing with Zero WTP Responses: Spike Model}

Some people may have a great interest in the good to be valued while others are totally indifferent to, or place no value on the good. In this case, the proportion of zero WTP responses in the CV survey may be high. Researchers should pay close attention to dealing with WTP responses with observations of zero. For this reason, we apply a spike model, as suggested by Kriström [29] and Yoo and Kwak [30]. The spike model specifies the probability of a WTP response of zero as a spike at zero in the distribution of WTP. The spike model enables us to analyze both point data at zero and positive interval WTP data in a univariate setting. In the spike model, $F_{X}(B ; \lambda)$ is assumed to have the following functional form:

$$
F_{X}(B ; \lambda)= \begin{cases}{[1+\exp (\alpha-\beta B)]^{-1}} & \text { if } B>0 \\ {[1+\exp (\alpha)]^{-1}} & \text { if } B=0 \\ 0 & \text { if } B<0\end{cases}
$$

As explained earlier, the spike is defined as the probability of the respondent's WTP being zero. Thus, the spike is computed as $[1+\exp (\alpha)]^{-1}$. Some covariates, such as the respondent's household income, can be incorporated into the spike model. A common method of doing this makes the covariates penetrate into $\alpha$ in Equation (7). That is, $\alpha$ is simply changed into $\alpha+z^{\prime} \delta$, where $z$ is a vector of covariates and $\delta$ is a vector of the corresponding parameters to be estimated.

\subsection{SB DC Spike Model}

As explained above, we use the SB DC model in this study. The following SB DC model is based on Hanemann's [26] suggestion. We have $T$ observations to be analyzed. A bid, $B_{j}$, is given to respondent $j$, for $j=1, \ldots, T$. There are two outcomes, "yes" $\left(Z \geq B_{j}\right)$ and "no" $\left(Z<B_{j}\right)$. Therefore, for the two results we can introduce two binary variables, $I_{j}^{Y}$ and $I_{j}^{N}$. The value of each binary variable is one if the respondent's response corresponds with its superscript, and zero otherwise. For example, $I_{j}^{Y}$ is one if respondent $j$ reports "yes", and zero otherwise.

We attempt to combine the SB DC CV model and the spike model. In order to identify the zero WTP observations, we asked the respondents who answered "no" to a presented bid $B_{j}$ an additional follow-up question to distinguish true zero WTP from positive WTP. Thus, we can define one more binary variable, $I_{j}^{P}$ whose value is one if respondent $j^{\prime}$ 's WTP is positive, and zero otherwise. The log-likelihood function of the SB DC spike model is:

$$
\begin{aligned}
& \ln L=\sum_{j=1}^{J}\left\{I_{j}^{Y} \ln \left[1-F_{X}\left(B_{j} ; \lambda\right)\right]+I_{j}^{P} I_{j}^{N} \ln \left[F_{X}\left(B_{j} ; \lambda\right)-F_{X}(0 ; \lambda)\right]+\right. \\
& \left.\left(1-I_{j}^{P}\right) I_{j}^{N} \ln F_{X}(0 ; \lambda)\right\}
\end{aligned}
$$

Using Equation (7), the mean of WTP can be computed as:

$$
E(X)=\int_{0}^{\infty}\left[1-F_{X}(B ; \alpha, \beta)\right] d B-\int_{-\infty}^{0} F_{X}(B ; \alpha, \beta) d B=\frac{1}{\beta} \ln [1+\exp (\alpha)]^{-1}
$$

\section{Empirical Results}

\subsection{Data}

The CV survey was administered to 560 randomly chosen households from the Seoul population during May 2013. Some observations that did not contain important information or were judged to be of poor quality by the interviewers were deleted from final dataset to be investigated. Thus, 
we obtained 500 useable observations. Table 1 describes the distribution of responses by bid amount. Each set of bids was allocated to a similar number of respondents, as is shown in the last column of Table 1.

Table 1. Distribution of responses by bid amount.

\begin{tabular}{ccccc}
\hline \multirow{2}{*}{ Bid Amount in KRW } & \multicolumn{3}{c}{ Number of Responses (\%) a } & \multirow{2}{*}{ Sample Size a } \\
\cline { 2 - 4 } & “Yes" & “No-Yes" & “No-No" & \\
\hline 1000 & $43(59.7)$ & $7(9.7)$ & $22(30.5)$ & $72(100.0)$ \\
2000 & $42(59.1)$ & $6(8.5)$ & $23(32.3)$ & $71(100.0)$ \\
3000 & $32(45.0)$ & $12(16.9)$ & $27(38.0)$ & $71(100.0)$ \\
5000 & $27(37.5)$ & $13(18.0)$ & $32(44.4)$ & $72(100.0)$ \\
7000 & $28(39.4)$ & $14(19.7)$ & $29(40.8)$ & $71(100.0)$ \\
10,000 & $20(28.1)$ & $17(23.9)$ & $34(47.8)$ & $71(100.0)$ \\
15,000 & $16(22.2)$ & $21(29.1)$ & $35(48.6)$ & $72(100.0)$ \\
Totals & $208(41.6)$ & $90(18.0)$ & $202(40.4)$ & $500(100.0)$ \\
\hline
\end{tabular}

Note: ${ }^{\text {a }}$ the numbers in parentheses beside the number of responses are the percentage of the sample size.

The "no-no" responses indicate the WTP responses of zero. A total of 202 households (40.4\%) revealed zero WTP for the transformation. This implies that the use of the spike model to deal with zero WTP responses was a suitable approach in our study. Moreover, a WTP response of zero is consistent with microeconomic theory that non-consumption can be obtained as a corner solution to a utility maximization problem under income constraint. Overall, the proportion of "yes" responses to a given bid declined as the magnitude of the bid increased. For instance, forty-three respondents $(59.7 \%)$ accepted the payment of KRW 1000 (USD 0.90), while just sixteen respondents (22.2\%) agreed to the payment of KRW 15,000 (USD 13.30).

In spite of an increase in respondent's property taxes, some respondents have positive WTP for the transformation. The reasons why they have positive WTP for the transformation through an increase in their property taxes are described in Table 2. For example, 152 respondents $(51.0 \%)$ reported positive WTP because they are likely to visit the JBT in the future although they have no plan to visit the JBT now.

Table 2. Reasons why a respondent has positive willingness to pay for the transformation.

\begin{tabular}{cc}
\hline Reasons & Number of Responses (\%) ${ }^{\text {a }}$ \\
\hline I want to visit the JBT in the near future. & $87(29.2)$ \\
Although I have no plan to visit the JBT now, I am likely to visit the JBT in the future. & $152(51.0)$ \\
I'm not likely to visit the JBT, but I support the transformation. & $59(19.8)$ \\
Totals & $298(100.0)$ \\
\hline
\end{tabular}

Note: ${ }^{a}$ the numbers in parentheses beside the number of responses are the percentage of the sample size.

\subsection{Estimation Results for the SB DC Spike Model}

The estimation results for the SB DC spike model with no covariates are reported in Table 3. The parameter estimates can be obtained by finding the parameter values maximizing Equation (8); that is, by applying the maximum likelihood estimation method. All of the estimates for the two parameters, $\alpha$ and $\beta$, are statistically significant at the $1 \%$ level. Moreover, the null hypothesis that the parameter estimates are all zero can be rejected at the $1 \%$ level, in that the $p$-value for the Wald statistic calculated under the null hypothesis is less than 0.01. In particular, the estimate for the spike is 0.4103 , which approximately coincides with the sample proportion of zero WTP responses provided in Table 1. This indicates that the spike model employed here fits our data well. 
Table 3. Estimation results for the spike model.

\begin{tabular}{|c|c|}
\hline Variables & Estimates $^{d}$ \\
\hline Constant & $0.3629(3.96)^{* *}$ \\
\hline $\mathrm{Bid}^{\mathrm{a}}$ & $-0.1250(-11.01) * *$ \\
\hline Spike & $0.4103(18.50)^{* *}$ \\
\hline Mean WTP per household per year & KRW 7129 (USD 6.30) \\
\hline$t$-value & $11.29 * *$ \\
\hline $95 \%$ confidence interval ${ }^{b}$ & KRW 6062 to 8601 (USD 5.40 to 7.60 ) \\
\hline $99 \%$ confidence interval $^{b}$ & KRW 5740 to 9132 (USD 5.10 to 8.10 ) \\
\hline Number of observations & 500 \\
\hline Log-likelihood & -510.93 \\
\hline Wald statistic $(p \text {-value })^{c}$ & $121.14(0.000)$ \\
\hline
\end{tabular}

Notes: ${ }^{a}$ the unit is 1000 Korean won; USD 1.00 was approximately equal to KRW 1131 at the time of the survey; $\mathrm{b}$ the confidence intervals are calculated by the use of the Monte Carlo simulation technique of Krinsky and Robb [31] with 5000 replications; ${ }^{c}$ the null hypothesis is that all the parameters are jointly zero and the corresponding $p$-value is reported in parentheses beside the statistic; ${ }^{\mathrm{d}}$ the numbers in parentheses beside the coefficient estimates are $t$-values, computed from the analytic second derivatives of the log-likelihood. ** indicates statistical significance at the $5 \%$ level.

Using Equation (9) and the values presented in Table 3 we can obtain an estimate of the mean WTP: KRW 7129 (USD 6.30) per household per year. Its $t$-value is 11.29 and, thus, the estimate is statistically meaningful at the $1 \%$ level. In order to handle the uncertainty related to the computation of the estimate, we try to report the confidence intervals for the estimate. For this purpose, the parametric bootstrapping method proposed by Krinsky and Robb [31] is the method most widely employed in the literature. Thus, we also use that method, with 5000 replications, to get the $95 \%$ and $99 \%$ confidence intervals, which are contained in Table 4 . The 95\% confidence interval is tighter than the $99 \%$ confidence interval.

Table 4. Definitions and sample statistics of the variables.

\begin{tabular}{clcc}
\hline Variables & \multicolumn{1}{c}{ Definitions } & Mean & Standard Deviation \\
\hline Value & $\begin{array}{l}\text { Subjective judgment about how the experience of } \\
\text { traditional culture is important (from 1 to 5) }\end{array}$ & 4.20 & 0.82 \\
\hline Education & The respondent's education level in years & 14.31 & 2.55 \\
\hline Visit & $\begin{array}{l}\text { The expected number of visits to JBT during the } \\
\text { five years after the project is finished }\end{array}$ & 3.90 & \multirow{2}{*}{3.22} \\
\hline Need & $\begin{array}{l}\text { Subjective judgment about the need to implement } \\
\text { the project (from 1 to 5) }\end{array}$ & 3.55 & \multirow{2}{*}{97.47} \\
\hline Income & $\begin{array}{l}\text { Household monthly income per capita } \\
\text { (unit: ten thousand Korean won = USD 8.84) }\end{array}$ & \\
\hline
\end{tabular}

\subsection{Estimation Results for the SB DC Spike Model with Covariates}

We seek to estimate the spike model with covariates explained in Section 3.2. Some variables used for the covariates are defined in Table 4. They are related to the characteristics of the respondent or the respondent's household. Furthermore, the sample statistics of the covariates are reported in Table 5. A total of five variables are contained in the model. The sample characteristics appear to reflect the population's characteristics well, since we drew a random sample of Seoul households with the help of the professional polling firm. The estimation results of the spike model including the variables presented in Table 4 are described in Table 5. 
Table 5. Estimation results of the spike model with covariates.

\begin{tabular}{ccc}
\hline Variables & Estimates & $t$-Values \\
\hline Constant & -2.6624 & $-3.55^{* *}$ \\
Bid $^{\text {a }}$ & -0.1513 & $-11.11^{* *}$ \\
Value & 0.1626 & 0.15 \\
Education & -0.0072 & -0.19 \\
Visit & 0.0918 & $6.88^{* *}$ \\
Need & 0.7095 & $6.81^{* *}$ \\
Income & 0.0018 & $1.70^{*}$ \\
Spike & 0.3894 & $16.46^{* *}$ \\
\hline Wald statistic $\left(p\right.$-value) $^{\mathrm{b}}$ & \multicolumn{2}{c}{$203.60(0.000)$} \\
Log-likelihood $_{\text {Number of observations }}$ & \multicolumn{2}{c}{500.07} \\
\hline
\end{tabular}

Notes: The variables are defined in Table $4 .{ }^{a}$ the unit is 1000 Korean won; USD 1.00 was approximately equal to KRW 1131 at the time of the survey; ${ }^{b}$ the null hypothesis is that all the parameters are jointly zero and the corresponding $p$-value is reported in parentheses beside the statistic. ${ }^{*}$ and ${ }^{* *}$ indicate statistical significance at the $10 \%$ and $5 \%$ levels, respectively.

In particular, the coefficient estimates for the Visit, Need, and Income variables in the model are statistically significant at the $10 \%$ level. This implies that a person who plans to visit JBT during the five years after the transformation is finished is more likely to accept a given bid for the implementation of the transformation than another person. Those who thought that the transformation was needed were more likely than others to answer "yes" to a presented bid. The finding that the estimated coefficient for Income has a positive sign indicates that respondents with a higher income have a tendency to respond "yes" to a given bid.

\subsection{Estimation of Total WTP}

In order to get an estimate of the total WTP, we use the mean WTP estimate obtained from the investigation of the sample observations and information on the population size. In the course of doing this, the most important issue was whether or not the sample was representative of the population. As addressed above, the sampling was conducted by a professional survey firm, to ensure the randomness of the sample and its consistency with the characteristics of the population. Another important issue is the response rate in the CV survey. Our CV survey was implemented using in-person face-to-face interviewing and thus the response rate was almost $100 \%$. Thus, we cannot deny that our sample was representative of the population.

We use the mean WTP estimate from the model with no covariates. This is because the setting of the covariates may influence the mean WTP value if we use the mean WTP estimate from the model with covariates. According to Statistics Korea (http:/ / kosis.kr) supplied by the Korea National Statistical Office, the number of households in Seoul was 3,567,727 (population was 9,926,383) in Seoul at the time of the survey, May 2013. Using this information, expanding the value to all Seoul citizens gives us KRW 25.4 billion (USD 22.5 million) per year. The present value of the total WTP amounts to KRW 114.6 billion (USD 101.3 million) using a social discount rate of $5.5 \%$. The corresponding $95 \%$ and 99\% confidence intervals for the total WTP are KRW 97.4 to 138.2 billion (USD 86.1 to 122.2 million) and KRW 92.3 to 146.8 billion (USD 81.6 to 129.8 million), respectively. Overall, we can conclude that Seoul households are ready to bear a share of the financial burden of implementing the transformation.

\section{Conclusions}

JBT is the chief temple of the Jogye Order, which represents Korean Buddhism. Despite this, the buildings where the stores and shops are located, and the environment surrounding JBT, are now old-fashioned and somewhat run-down. Thus, the Seoul government plans to implement a project to transform JBT into a cultural tourism resource and historical site. More specifically, the plans 
being considered are to build a new shopping arcade for Buddhist culture and tourism, to construct a museum for teaching history (the 10.27 event) and an experience center for Korean traditional culture in the precincts of JBT, and to make an open space for domestic and/or foreign visitors. Since the cost of the transformation will be met by local taxes, public support for the transformation is quite important.

Therefore, the Seoul government asked for quantitative information on the WTP for implementing the transformation. The eventual objective of the transformation is to make JBT a good precedent which is referred by cultural policy-makers. Furthermore, the transformation can serve as a momentum, which means that Korea cultural heritages of religion can be developed as tourism resources in the near future. The demand of sustainable tourism resources has become an important aspect that must be considered by local government [32]. The transformation has significant meaning as sustainability of the excavation of tourism resources. In this regard, we attempted to report the findings from a CV survey that measures the WTP arising from the implementation of the transformation.

Based on the interviewers' comments, the value judgments the respondents were required to make were within their abilities. Moreover, all of the parameter estimates from the model have high statistical significance. Thus, the estimated SB DC spike model is statistically meaningful. We found that the mean WTP estimate for the implementation of the transformation is KRW 7129 (USD 6.30) per household per year for the next five years, and that this estimate has high statistical significance. Expanding the value to the city's whole population gave us KRW 25.4 billion (USD 22.5 million) per year, and the present value of the transformation was computed to be KRW 114.6 billion (USD 101.3 million). We can conclude that Seoul households are ready to shoulder some of the financial burden of implementing the transformation.

Policy implications of whether to implement the transformation or not, could, in principle, be deduced from an examination of costs and benefits associated with the transformation. An important first step in fostering a productive public debate on the transformation is a better understanding of its benefits and costs. The costs of implementing the transformation can be relatively easily computed. On the other hand, measuring the benefits expected from the transformation is quite a difficulty work. The benefits can be compared with the costs of the transformation to determine whether the transformation is economically desirable and whether the decision to implement the transformation should be made. Our study provided information on the public value of the transformation, which can be added to the private value of the transformation to obtain a figure about the economic benefits of the transformation. Moreover, the framework and results of this study can be referred to when other similar programs are being designed in Seoul. We can guess that the public value of these programs are close to the values obtained in this study, although an empirical study valuing the programs should be done to get accurate value information.

As a second step of the study, the following topic should be examined in a future study. In particular, the focus of our study is placed only on the public value or public WTP. However, the transformation is also likely to generate some environmental or congestion costs. This issue has been frequently discussed in the tourism literature. For example, Marsiglio [33] discussed how the WTP in a tourism context might depend on several factors, including environmental quality and congestion factors). The eventual direct or indirect costs associated with the transformation should be taken into account. Marsiglio [33] and Farr et al. [34] dealt with the issue from a theoretical point of view and from an empirical one, respectively. Although our dataset cannot allow us to analyze the issue, it should be noted that these factors is likely to affect and distort the results.

Author Contributions: Seul-Ye Lim designed the ideas and analyzed the data. Ho-Young Kim wrote the first draft manuscript. Seung-Hoon Yoo improved the first draft and took the correspondence. With different contributions, all the co-authors enriched the submitted manuscript and ended it up with the current publication.

Conflicts of Interest: The Seoul government financially supported this study. However, the authors declare they have no conflicts of interest. 


\section{References}

1. Cho, F. Buddhist mind and matter. Religions 2014, 5, 422-434. [CrossRef]

2. Samuel, G. Between buddhism and science, between mind and body. Religions 2014, 5, 560-579. [CrossRef]

3. Jogye Order of Korean Buddhism. Available online: http://www.jogyesa.kr/user/jogye (accessed on 14 September 2015). (In Korean)

4. Visit Seoul Net. Available online: http://english.visitseoul.net/attractions/Jogyesa-Temple_/2586 (accessed on 14 September 2015). (In Korean)

5. Visit Korea. Available online: http://korean.visitkorea.or.kr/kor/bz15/where/where_main_search.jsp?cid= 128144 (accessed on 14 September 2015). (In Korean)

6. Seoul Metropolitan Government. Available online: http://opengov.seoul.go.kr/scholarship/410050 (accessed on 14 September 2015). (In Korean)

7. Brent, R.J. Applied Cost-Benefit Analysis; Cheltenham: Edward Elgar, UK, 1995.

8. Huang, C.-H.; Wang, C.-H. Estimating the total economic value of cultivated flower land in Taiwan. Sustainability 2015, 7, 4764-4782. [CrossRef]

9. Kwak, S.-Y.; Yoo, S.-H. The public value of a national library: Results of a contingent valuation survey. J. Librariansh. Inf. Sci. 2012, 44, 263-271. [CrossRef]

10. Choi, H.-Y.; Kwak, S.-J.; Yoo, S.-H. The preservation value of Bangudae petroglyphs: The 285th Korean national treasure. J. Cult. Herit. 2016, 18, 380-383. [CrossRef]

11. Miccoli, S.; Finucci, F.; Murro, R. Social evaluation approaches in landscape projects. Sustainability 2014, 6, 7906-7920. [CrossRef]

12. Pérez-Álvarez, R.; Torres-Ortega, S.; Díaz-Simal, P.; Husillos-Rodríguez, R.; de Luis-Ruiz, J.M. Economic valuation of mining heritage from a recreational approach: application to the case of el soplao cave in Spain (Geosite UR004). Sustainability 2016, 8, 185. [CrossRef]

13. Harun, R.; Muresan, I.C.; Arion, F.H.; Dumitras, D.E.; Lile, R. Analysis of factors that influence the willingness to pay for irrigation water in the Kurdistan regional government, Iraq. Sustainability 2015, 7, 9574-9586. [CrossRef]

14. Loures, L.; Loures, A.; Nunes, J.; Panagopoulos, T. Landscape valuation of environmental amenities throughout the application of direct and indirect methods. Sustainability 2015, 7, 794-810. [CrossRef]

15. Hanley, N.; Barbier, E.B. Pricing Nature: Cost-Benefit Analysis and Environmental Policy; Cheltenham: Edward Elgar, UK, 2009.

16. Mitchell, R.C.; Carson, R.T. Using Surveys to Value Public Goods: The Contingent Valuation Method; Resources for the Future: Washington, DC, USA, 1989.

17. Kwak, S.J.; Yoo, S.H.; Lee, C.K. Valuation of the woopo wetland in Korea: A contingent valuation study. Environ. Dev. Econ. 2007, 12, 323-328. [CrossRef]

18. Yoo, S.H.; Kwak, S.Y. Willingness to pay for green electricity in Korea. Energy Policy 2009, 37, 5408-5416. [CrossRef]

19. Lee, J.S.; Yoo, S.H. Willingness to pay for GMO labeling policies: The case of Korea. J. Food Saf. 2011, 30, 160-168. [CrossRef]

20. Lee, W.-S.; Yoo, S.-H.; Kim, J. Measuring the economic benefits of the tap water supply service in urban areas: The case of Korea. Water Resour. Manag. 2013, 27, 619-627. [CrossRef]

21. Arrow, K.; Solow, R.; Portney, P.R.; Leamer, E.E.; Radner, R.; Schuman, H. Report of the NOAA panel on contingent valuation. Fed. Regist. 1993, 58, 4601-4614.

22. Hanemann, W.M.; Loomis, J.; Kanninen, B.J. Statistical efficiency of double-bounded dichotomous choice contingent valuation. Am. J. Agric. Econ. 1991, 73, 1255-1263. [CrossRef]

23. Bateman, I.J.; Langford, L.H.; Jones, P.; Kerr, G.N. Bound and path effects in double and triple bounded dichotomous choice contingent valuation. Resour. Energy Econ. 2001, 23, 191-213. [CrossRef]

24. Carson, R.T.; Groves, T. Incentive and informational properties of preference questions. Environ. Resour. Econ. 2007, 37, 181-210. [CrossRef]

25. Egan, K.J.; Corrigan, J.R.; Dwyer, D.F. Three reasons to use annual payments in contingent valuation surveys: Convergent validity, discount rates, and mental accounting. J. Environ. Econ. Manag. 2015, 72, 123-136. [CrossRef] 
26. Hanemann, W.M. Welfare evaluations in contingent valuation experiments with discrete responses. Am. J. Agric. Econ. 1984, 66, 332-341. [CrossRef]

27. Cameron, T.A.; James, M.D. Efficient estimation methods for "closed-ended" contingent valuation surveys. Rev. Econ. Stat. 1987, 69, 269-276. [CrossRef]

28. McConnell, K.E. Models for referendum data: The structure of discrete choice models for contingent valuation. J. Environ. Econ. Manag. 1990, 18, 19-34. [CrossRef]

29. Kriström, B. Spike models in contingent valuation. Am. J. Agric. Econ. 1997, 79, 1013-1023. [CrossRef]

30. Yoo, S.H.; Kwak, S.J. Using a spike model to deal with zero response data from double bounded dichotomous choice contingent valuation surveys. Appl. Econ. Lett. 2002, 9, 929-932. [CrossRef]

31. Krinsky, L.; Robb, A. On approximating the statistical properties of elasticities. Rev. Econ. Stat. 1986, 68, 715-719. [CrossRef]

32. Rahauningsih, T.; Muntasib, E.K.S.H.; Prasetyo, L.B. Nature based tourism resources assessment using geographic information system (GIS): Case study in Bogor. Procedia Environ. Sci. 2016, 33, 365-375. [CrossRef]

33. Marsiglio, S. Economic growth and environment: Tourism as a trigger for green growth. Tour. Econ. 2015, 21, 183-204. [CrossRef]

34. Farr, M.; Stoeckl, N.; Esparon, M.; Larson, S.; Jarvis, D. The importance of water clarity to tourists in the Great Barrier Reef and their willingness to pay to improve it. Tour. Econ. 2016, 22, 331-352.

(C) 2016 by the authors; licensee MDPI, Basel, Switzerland. This article is an open access article distributed under the terms and conditions of the Creative Commons Attribution (CC-BY) license (http:/ / creativecommons.org/licenses/by/4.0/). 